\title{
On Conformal Representation of Multiply Connected Polygonal Domain.
}

\author{
Akira Mori.
}

(Received Oct. 1, 1949)

It is known, that a function $z(z)$ is schlicht and star-shaped with respect to $w(0)=0$ in $|z|<1$, when, and only when, it can be expressed in the form

$$
w(z)=\text { const. } \cdot z \cdot \exp .2 \int_{|\zeta|=1} \log \frac{\zeta}{\zeta-z} d \mu(\zeta),
$$

where $\mu$ denotes a positive distribution of total mass 1 on the unit circle. This formula can also be written in the form

$$
w(z)=\text { const. } \exp \cdot \int_{|\zeta|=1} \log \frac{z}{\left(1-\frac{z}{\zeta}\right)^{2}} d \mu(\zeta),
$$

and here comes out Koebe's extremal function. The argument of this function is equal to a constant on $|z|=1$ except the point $\zeta$, and jumps by $+2 \pi$ when $z$ passes $\zeta$ in positive direction on $|z|=1$. Then, the above formula shows: The star-shaped function $w(z)$, whose argument is nondecreasing for $z$ moving on $|z|=1$ in positive direction, can be constructed from such clements as a sort of geometrical mean.

We shall prove in this paper an analogue of this fact for $n$-ply connected domain, and, as an application thereof, treat the conformal representation of $n$-ply connected polygonal domain.

In order to simplify the wording, we call a half straight-line Arg $\boldsymbol{\Omega}=$ const., $|\boldsymbol{\Omega}| \geqq$ const. $>0$ an "infinite radial slit", and a segment $\operatorname{Arg} \Omega=$ const., const. $\geqq|\Omega| \geqq$ const. $>0$ a "radial slit", respectively.

\section{$\S 1$.}

Let $D$ be a domain on $z$-plane bounded by $n$ analytic closed curves $\Gamma_{1}, \cdots \cdots, \Gamma_{n}$, whose sum we denote by $\Gamma$, and let $z_{0}$ be a fixed point in $D$.

For any point $\zeta$ on $\Gamma$, we denote by $\Omega(z, \zeta)$ the function which satisfies the conditions $\Omega\left(z_{0}, \zeta\right)=0, \Omega \Omega^{\prime}\left(z_{0}, \zeta\right)=1$ and maps $D$ conformally on the whole $\Omega$-plane cut along an infinite radial slit and $(n-1)$ radial slits, so that the boundary point $\zeta$ of $D$ corresponds to the bodunary point 
$\boldsymbol{\Omega}=\infty$. The existence, uniqueness and the continuity in $\boldsymbol{\zeta}$ of such functions are to be proved afterwards in Lemmas 1 . and 3 .

We will now formulate the theorem to be proved as follows :

Theorem 1. Lot $w(\approx)$ be a function which satisfies the following three conditions :

1. $w(z)$ is regular and does not vanish in $D$ except at $z_{0}$, where it has an expansion of the form

$$
w(z)=\left(z-z_{0}\right)^{\alpha}\left\{1+c_{1}\left(z-z_{0}\right)+\cdots \cdots\right\} \quad(\alpha \geqq 0) .
$$

2. $|w(\dot{v})|$ is one-valued in 1$)$.

3. Any branch of Arg $w(z)$ is bounded in the neighbourhood of $\Gamma$, and the limiting value

$$
\lim _{z \rightarrow \zeta^{*}} \operatorname{Arg} w(z)=\theta\left(\zeta^{*}\right)
$$

exists for each $\zeta^{*}$ on $\Gamma$ except at most an enumerable infinity of points and is of bounded variation, as function of $\zeta^{*}$ on $\Gamma$, on the set where it exists.

$A$ necessary and sufficient condition for this, is that $w(z)$ can be expressed in the form

$$
w(z)=\exp \cdot \int_{\Gamma} \log \Omega(z, \zeta) d \sigma(\zeta),
$$

where $\sigma$ is a distribution of bounded variation of total mass $\alpha$ on $\Gamma$, determined by the function of bounded variation $\frac{1}{2 \pi} \theta\left(\zeta^{*}\right)$.

We shall make some preparations and prove some lemmas.

Definition of the Riemann surface $\Phi$. Let $\tilde{D}$ be another sheet of $D$. We put $D^{\prime}$ on $D$ and identify the corresponding boundary points of $D$ and $\tilde{D .}$ This closed surface can be regarded as a closed Riemann surface $\Phi$ of genus $n-1$, since we can define a local parameter $t(p)$ for each point $p$ on $\Phi$ : for a point of $\tilde{D}$ by taking conjugate complex, and for a point on $\Gamma$ by reflection in $\Gamma$.

By interchanging the two sheets $D$ and $\tilde{D}$, we obtain a transformation $p \rightarrow \tilde{p}$ which transforms $\Phi$ into itself conformally with inversion of angles.

Besides, we denote by $\omega_{q_{1} q_{2}}(p)$ the elementary integral of third kind on $\Phi$, which has the singularities $\log t\left(q_{1}\right)$ at $q_{1}$ and $-\log t\left(q_{2}\right)$ at $q_{2}$, and whose real part is one-valued on $\Phi$. And by $\omega_{q_{1}, q_{2}}^{\prime}(p)$ we denote the elementary integral of third kind, which has the singularities $-i \log t\left(q_{1}\right)$ at 
On conformal representation of multiply connected polygonal domain. 189 $q_{1}$ and $i \log t\left(q_{2}\right)$ at $q_{2}$, and whose real part is one-valued on $\Phi$ cut along a curve connecting $q_{1}$ with $q_{2}^{1)}$.

Lemma 1. For each $\zeta$ on $\Gamma$, there exists one and only one function $\boldsymbol{\Omega}(z, \zeta)$ with the mentioned properties.

Proof. We put.

$$
\omega_{z_{0}, \xi}(p)+\omega_{\tilde{z_{0}} \xi}(p)=u(p)+i v(p),
$$

and

$$
\Omega(z, \zeta)=\text { const. exp. }\{u(z)+i v(z)\}
$$

Since the one-valued potential function $u(\tilde{p})$ has on $\Phi$ the same singularities as $u(p)$, and takes on $\Gamma$ the same value as $u(p)$, we have

$$
u(p) \equiv u(\tilde{p}),
$$

i.e. $u(p)$ takes the same value at $\tilde{p}$ as at $p$. Hence we have at each point on $\Gamma$ except $\zeta$,

$$
\frac{\partial u}{\partial \nu}=0 \quad \text { consequently } \frac{\partial v}{\partial \tau}=0,
$$

where $\nu$ and $\tau$ denote the normal and tangent to $\Gamma$. Therefore, $v$ takes a constant value on each $\Gamma_{k}$. It follows from this, that $\Omega(z, \zeta)$ is onevalued in $D$.

On the other hand, $u$ is finite at each point of $\Gamma$ except $\zeta$, where $u$ is positively infinite. Hence, the image of $\Gamma^{\prime}$ by $\Omega(z, \zeta)$ consists of an infinite radial slit and $n-1$ radial slits.

Let $\Omega_{0}$ be a point of $\boldsymbol{\Omega}$-plane, which does not belong to these $n$ slits. Since

$$
\operatorname{Arg}\left\{\frac{1}{\Omega(z, \zeta)}-\frac{1}{\Omega_{0}}\right\}
$$

remains unchanged when $z$ moves on $\Gamma$ once around and returns to the original value, and since $1 / \Omega(z, \zeta)$ has one and only one pole in $D$, $\boldsymbol{\Omega}(z, \zeta)$ takes each value $\Omega_{0}$ once and only once in $D$. Therefore, $\boldsymbol{\Omega}(z, \zeta)$ provides the required mapping, when the constant factor in (2) is determined by the condition $\Omega^{\prime}\left(z_{0}, \zeta\right)=1$.

The uniqueness of the mapping function can be proved as follows. Let $\Omega_{1}(z, \zeta)$ be another mapping function with the mentioned properties. When 
we continue $\Omega_{1}(z, \zeta)$ analytically across $I^{\prime}$ on $\Phi$ by the principle of reflection, we obtain a one-valued potential function $\log \left|\Omega_{1}(p, \zeta)\right|$ on $\Phi$, since $\left|\Omega_{1}\right|$ remains unchanged by reflection in a radial slit. Moreover $\log \left|\Omega_{1}(p, \zeta)\right|$ has the same singularities as $u(p)$. Therefore, by the normalisation $\Omega_{1}^{\prime}\left(z_{0}, \zeta\right)=1, \Omega_{1}(z, \zeta)$ must be identical with $\Omega(z, \zeta)$.

Remark. By the same idea as in the above proof, we can construct the function $\Omega\left(z, z^{*}\right)$ for $z^{*} \in D$, which maps $D$ conformally on the whole $\boldsymbol{\Omega}$-plane cut along $n$ radial slits and satisfies $\boldsymbol{\Omega}\left(z_{0}, z^{*}\right)=0, \Omega^{\prime}\left(z_{0}, z^{*}\right)=1$, $\boldsymbol{\Omega}\left(z^{*}, z^{*}\right)=\infty$. For this purpose, we have only to put

$$
\boldsymbol{Q}\left(z, z^{*}\right)=\text { const. exp. }\left\{\omega_{z_{0} *}(z)+\omega_{\tilde{z}, \tilde{z}} \tilde{z}(z)\right\} \text {. }
$$

We cut the domain $D$ by $n$ curves, each of which connects $z_{0}$ respectively with an arbitrarily fixed point $\zeta_{k}$ on $\Gamma_{k}$, and which do not cross each others. We denote by $D_{0}$ the resulting simply connected domain, in which $\operatorname{Arg} \boldsymbol{Q}(z, \zeta)$ is one-valued. We can assume that $D_{0}$ contains wholly in it a line element $d x$ at $z_{0}$ with direction of positive real axis. We take the branch of $\operatorname{Arg} Q(z, \zeta)$ which vanishes at $z_{0}+d x$ and put

for $z \in D_{0}$.

$$
\boldsymbol{\theta}(z, \zeta)=\operatorname{Arg} \boldsymbol{\Omega}(z, \zeta)
$$

As function of $z$ with fixed $\zeta, \theta(z, \zeta)$ has the following properties.

Lemma 2. $\theta(z, \zeta)$ is bounded in $D_{0}$, and the limiting value

$$
\lim _{z \rightarrow \zeta^{*}} \theta(z, \zeta)=\theta\left(\zeta^{*}, \zeta\right)
$$

exists for each $\zeta^{*}$ on $\Gamma$ except $\zeta . \quad \theta\left(\zeta^{*}, \zeta\right)$ is equal to a constant on each arc of $\Gamma$ which contains neither $\zeta$ nor $\zeta_{1} \cdots \cdots \zeta_{n}$, and jumps by $+2 \pi$ at $\zeta$ when $\zeta^{*}$ moves on $I$ in pocitive direction.

Proof. This is obvious from the shape of the image of $D$ by $\Omega(z, \zeta)$.

As function of $\zeta$ with fixed $z, \log \Omega(z, \zeta)$ and $\theta(z, \zeta)$ have the following properties.

Lemma 3. $\log \Omega(z, \zeta)$ is one-valued and continuous, and its imaginary part $\theta(z, \zeta)$ is uniformly bounded for the parameter $z$ in $D_{0}$.

Proof. The constant factor in (2), which is to be determined by the condition $\Omega^{\prime}\left(z_{0}, \zeta\right)=1$, depends naturally on $\zeta$. When we write (2) in the form

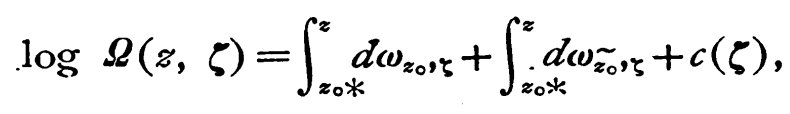

where $z_{0}{ }^{*}$ denotes an arbitrarily fixed point in $D$, the condition $\mathscr{Q}^{\prime}\left(z_{0}, \zeta\right)=1$ 
On conformal representation of multiply connected.polygonal domain. 191 is given by

$$
c(\zeta)=\lim _{z_{1 \rightarrow z_{0}}}\left\{-\int_{z_{0} 0^{*}}^{z_{1}} d\left(\omega_{Z_{0}, \zeta}-\int_{z_{0}^{* *}}^{z_{1}} d\left(\omega_{z_{0}, \zeta}+\log \left(z_{1}-z_{0}\right),\right\},\right.\right.
$$

and we obtain the following definite form of $\log \Omega(z, \zeta)$,

$$
\log \Omega(z, \zeta)=\lim _{z_{1} \rightarrow z_{0}}\left\{\int_{z_{1}}^{z} d \omega_{z_{0}}{ }_{\zeta}+\int_{z_{1}}^{z} d \omega_{z_{0}, \zeta}+\log \left(z_{1}-z_{0}\right)\right\} .
$$

We assume that $\zeta$ lies on $\Gamma_{k}$, and consider the difference

$$
\begin{aligned}
& \log \Omega(z, \zeta)-\log \Omega\left(z, \zeta_{k}\right) \\
& =\lim _{z_{1} \rightarrow z_{0}}\left\{\int_{z_{1}}^{z} d \omega_{z_{0}, \zeta}-\int_{z_{1}}^{z} d \omega_{Z_{0}, \zeta_{k}}+\int_{z_{1}}^{z} d \omega_{z_{0}, \zeta}-\int_{z_{1}}^{z} d \omega_{z_{0}, \zeta_{k}}\right\} \\
& =\lim _{z_{1} \rightarrow z_{0}} 2 \int_{z_{1}}^{z} d \omega_{\zeta_{k}, \zeta}=2 \int_{z_{0}}^{z} d \omega_{\zeta_{k}, \zeta .}
\end{aligned}
$$

By the theorem of interchange of argument and parameter, ${ }^{2)}$ we can write this in the form

$$
\log \Omega(z, \zeta)-\log \Omega\left(z, \zeta_{k}\right)=2\left\{\Re \int_{\zeta_{k}}^{\zeta} d \omega_{z_{0}, z}+i \Re \int_{\zeta_{k}}^{\zeta} d \omega^{\prime}{ }_{z_{0} \prime z}\right\} .
$$

This proves the mentioned property of $\log \Omega(z, \zeta)$.

While taking the imaginary part of this formula, we have

$$
\Theta(z, \zeta)-\Theta\left(z, \zeta_{k}\right)=2 \Re \int_{\zeta_{k}}^{\zeta} d \omega^{\prime} z_{0, z}
$$

Since the right-hand side is certainly uniformly bounded for $z$ in $\dot{D}_{0}$, and since $\theta\left(z, \zeta_{k}\right)$ is, by Lemma 2 , bounded in $D_{0}, \theta(z, \zeta)$ is uniformly bounded for $z$ in $D_{0}$.

Lemma 4. Let $f(z)$ be a function one-valued and regular in $D$, whose imaginary part is bounded. If the limiting value

$$
\lim _{z \rightarrow \zeta} \mathfrak{J} f(z)
$$

exists for each $\zeta$ on $\Gamma$ except at most an enumerable infinity of points, and if this limiting value is equal respectively to a constant on each $\Gamma_{k}$, then $f(z)$ is identically equal to a constant.

Proof. In the first place, since $\mathfrak{\Im} f(z)$ is bounded, there exist in fact no exceptional points. Then, we can continue $f(z)$ analytically on $\Phi$ across each $\Gamma_{k}$, by the principle of reflection. Since $\Re f(z)$ remains unchanged by 
reflection in a straight-line parallel to the real axis, we obtain, by this continuation, a one-valued potential function $\Re f(p)$ everywhere regular on $\Phi$, which must be identically a constant.

$$
\text { Q. E. D. }
$$

Now we will prove Theorem 1.

\section{Proof of Theorem 1.}

Sufficiency. Since $\log \Omega(z, \zeta)$ is continuous as function of $\zeta$ by Lemma 3 ,

$$
w(z)=\exp \cdot \int_{\mathbf{r}} \boldsymbol{Q}(z, \zeta) d \sigma(\zeta)
$$

represents an analytic function of $z$, which obviously satisfies the conditions 1 and 2 The property 3 can be proved as follows.

For a branch of Arg $w(z)$ one-valued in $D_{0}$, we have

$$
\operatorname{Arg} w(z)=\int_{\Gamma} \theta(z, \zeta) d \sigma(\zeta)
$$

By Lemma 3, this function of $z$ is bounded in $D_{0}$. When $z$ approaches to a point $\zeta^{*}$ on $\Gamma$, which is a point of continuity of the distribution $\sigma$, we have, by Lemmas 2,3 and by Lebesgue's theorem,

$$
\theta\left(\zeta^{*}\right)=\lim _{z \rightarrow \zeta^{*}} \operatorname{Arg} z(z)=\lim _{z \rightarrow \zeta^{*}} \int_{\Gamma} \theta(z, \zeta) d \sigma(\zeta)=\int_{\Gamma} \theta\left(\zeta^{*}, \zeta\right) d \sigma(\zeta) .
$$

Therefore, the limiting value $\theta\left(\zeta^{*}\right)$ certainly exists for a point of continuity of $\sigma$.

Let $C$ be an arc of positive direction on $I_{k}^{\circ}$, which does not contain the point $\zeta_{h}$, and whose starting and ending points $\zeta_{1}{ }^{*}, \zeta_{2}{ }^{*}$ are both points of continuity of $\sigma$.

Then, we have

$$
\theta\left(\zeta_{2}^{*}\right)-\theta\left(\zeta_{1}^{*}\right)=\int_{\Gamma}\left\{\theta\left(\zeta_{2}^{*}, \zeta\right)-\theta\left(\zeta_{1}^{*}, \zeta\right)\right\} d \sigma(\zeta)
$$

On the other hand, we have by Lemma 2,

$$
\theta\left(\zeta_{2}^{*}, \zeta\right)-\theta\left(\zeta_{1}^{*}, \zeta\right)=\left\{\begin{array}{llll}
2 \pi & \zeta \in C, \\
0 & \zeta \notin C .
\end{array}\right.
$$

Therefore we obtain

$$
\theta\left(\zeta_{2}^{*}\right)-\theta\left(\zeta_{1}^{*}\right)=2 \pi \sigma(C)
$$


On conformal representation of multiply connected polygonal domain. 193 and $\sigma$.

This proves the last part of 3 and the mentioned relation between $\theta$ Necessity. Let $w(z)$ be a function which satisfies the conditions 1, 2 and 3. We define by $\frac{1}{2 \pi} \theta\left(\zeta^{*}\right)$ a distribution $\sigma$ of bounded variation on $\Gamma$. Obviously $\sigma$ has the total mass $\alpha$. We put

and

$$
\begin{aligned}
w_{1}(z) & =\exp \cdot \int_{\Gamma} \log \Omega(z, \zeta) d \sigma(\zeta), \\
\theta_{1}\left(\zeta^{*}\right) & =\lim _{z \rightarrow \zeta^{* *}} \operatorname{Arg} w_{1}(z) \\
f(z) & =\log _{\frac{w}{w}(z)} .
\end{aligned}
$$

$f(z)$ is one-valued and regular in $D$.

Let $C$ be such an arc of $\Gamma_{k}$, as mentioned in the first part of this proof. Then we have

$$
\begin{aligned}
& \lim _{z \rightarrow \zeta_{2}^{* *}} \mathfrak{J} f(z)-\lim _{z \rightarrow \zeta_{1^{*}}} \mathfrak{I} f(z) \\
& =\left\{\theta\left(\zeta_{2^{*}}^{*}\right)-\theta_{1}\left(\zeta_{2^{*}}{ }^{*}\right)\right\}-\left\{\theta\left(\zeta_{1}^{*}\right)-\theta_{1}\left(\zeta_{1}^{*}\right)\right\} \\
& =\left\{\theta\left(\zeta_{2}^{*}\right)-\theta\left(\zeta_{1}^{*}\right)\right\}-\left\{\theta_{1}\left(\zeta_{2}^{*}\right)-\theta_{1}\left(\zeta_{1}^{*}\right)\right\} \\
& =2 \pi \sigma(C)-2 \pi \sigma(C)=0 .
\end{aligned}
$$

Therefore, $\Im f(z)$ has a constant limiting value on each $\Gamma_{k}$ respectively. Further, $\Im f(z)$ is bounded in $D$, since $\operatorname{Arg} w(z)$ and $\operatorname{Arg} w_{1}(z)$ are both bounded in $D_{0}$. Consequently by Lemma 4 we obtain

$$
f(z) \equiv \text { const., }
$$

and the normalisation in condition 1 gives

$$
v(z) \equiv w_{1}(z) \text {. }
$$

Q. E. D.

Remark 2 Making use of conformal representation, Theorem 1 finds itself valid, in the form as it stands, for any $n$-ply connected Jordan domain.

\section{$\S 2$.}

If $D$ is the interior of a circle or a circular ring-shaped domain, we can write down the explicit forms of $\Omega(z, \zeta)$ and $\Omega\left(z, z^{*}\right) .^{3)}$ 
The case where $D$ is the interior of the unit circle $|z|<1$ and $z_{0}$ is the origin $z=0$.

By reflection in $|z|=1$, the Riemann surface $\Phi$ represents itself conformally on the whole $z$-plane. And the elementary integral $z_{z 0, z_{1}}$ is given by

$$
w_{z_{0}, z_{1}}(z)=\log \frac{z}{z-z_{1}}+\text { const.. }
$$

Wnile giving suitable values to $z_{1}$ and combining them, we obtain by (2) and (3)

and

$$
\Omega(z, \zeta)=\frac{z}{\left(1-\frac{z}{\zeta}\right)^{2}}
$$

$$
\Omega\left(z, z^{*}\right)=\frac{z}{\left(1-\frac{z}{z^{*}}\right)\left(1-\bar{z}^{*} z\right)}
$$

under consideration of the normalisation $\Omega^{\prime}(0)=1$.

The case where $D$ is the ring-shaped domain $q<|z|<1$ and $z_{0}$ is real and positive. tion

By repeated reflections in the boundary curves, and by the transforma-

$$
u=u(z)=-i \log \frac{z}{z_{0}}
$$

the universal covering surface of $\Phi$ is mapped conformally on the whole finite $u$-plane. Then, putting $u_{1}=u\left(z_{1}\right), \omega_{z_{0}, z_{1}}$ is given by

$$
\omega_{z_{0}, z_{1}}=\log \frac{\sigma(u)}{\sigma\left(u-u_{1}\right)}-\left(\frac{\eta_{1}}{\omega_{1}} \Re u_{1}+i \frac{\eta_{3}}{\omega_{3}} \Im u_{1}\right) u+\text { const., }
$$

where $\sigma$ denotes the Weierstrass' $\sigma$-function with primitive periods

$$
2 \omega_{1}=2 \pi, 2 \omega_{3}=-2 i \log q
$$

and $\eta_{1}$ and $\eta_{3}$ have the ordinary significations.

While giving suitable values to $z_{1}$ and combining them, we obtain by (3), after simple calculations,

$$
\begin{aligned}
& \boldsymbol{S}\left(z, z^{*}\right)= \\
& -i z_{0} \frac{\dot{\sigma}\left(i \log \frac{z_{0}}{z^{*}}\right) \sigma\left(i \log \bar{z}^{*} z_{0}\right)}{\sigma\left(2 i \log z_{0}\right)} \cdot \frac{\sigma\left(i \log \frac{z}{z_{0}}\right) \sigma\left(i \log z_{0}^{\prime} z\right)}{\sigma\left(i \log \frac{z}{z^{*}}\right) \sigma\left(i \log \bar{z}^{*} z\right)} \cdot\left(\frac{z}{z_{0}}\right)^{2 i \frac{\eta_{1}}{\pi} \operatorname{Arg} z^{*}}
\end{aligned}
$$


On conformal representation of multiply connected polygonal domain. 195

under consideration of the normalisation $\Omega^{\prime}\left(z_{0}\right)=1$.

When we replace $z^{*}$ by $\zeta$ in the above formula, we obtain the expression for $\Omega(z, \zeta)$. But it can be a little simplified by separating the two cases $|\zeta|=1$ and $|\zeta|=q$. In fact, we have

$$
\begin{aligned}
& \Omega\left(z, e^{i \varphi}\right)= \\
& -\frac{i z_{0}}{\sigma\left(2 i \log z_{0}\right)} \cdot \frac{\sigma\left(i \log z_{0}+\varphi\right)^{2}}{\sigma(i \log z+\varphi)^{2}} \cdot \sigma\left(i \log \frac{z}{z_{0}}\right) \sigma\left(i \log z_{0} z\right) \cdot\left(\frac{z}{z_{0}}\right)^{2 i \frac{\eta_{1}}{\pi} \varphi}
\end{aligned}
$$

and

$$
\begin{aligned}
& \Omega\left(z, q e^{z \varphi}\right)= \\
& -\frac{i z_{0}}{\sigma\left(2 i \log z_{0}\right)} \cdot \frac{\sigma_{3}\left(i \log z_{0}+\varphi\right)^{2}}{\sigma_{3}(i \log z+\varphi)^{2}} \cdot \sigma\left(i \log \frac{z}{z_{0}}\right) \sigma\left(i \log z_{0} z\right) \cdot\left(\frac{z}{z_{0}}\right)^{2 i \frac{\eta_{1}}{\pi} \varphi}
\end{aligned}
$$

Remark. If $D$ is the domain $|z|<1$, or if $D$ is $q<|z|<1$ and $\alpha=0$, while differentiating the logarithm of (1) and multiplying it by $z$, we obtain by the above expressions for $\Omega(z, \zeta)$ 'the Poisson-Stieltjes' or the Villat-Stieltjes ${ }^{\prime 4)}$ expression for $z w^{\prime} / z e$. Further, it is easy to prove from Theorem 1 these two formulae in their perfect forms.

\section{$\S 3$.}

As an application of Theorem 1, we shall give an expression for the mapping function of $n$-ply connected polygonal domain, an analogue of Schwarz-Christoffel's formula. Here, by the word " $n$-ply connected polygonal domain", we mean an n-ply connected Riemann surface $P$ of planar character (schlichtartig), whose boundary consists of a finite number of segments or half straight-lines. $P$ may contain in it a finite number of points of ramification, and may cover the point at infinity a finite number of times.

We assume that $n$ is greater than 1 . Let $D$ be a concentric circular ring $R_{1}<|z|<R_{2}$ with $n-2$ concentric circular slits, whose $2(n-2)$ end points we denote by $s_{k}(k=1, \cdots \cdots)$. And we fix a point $z_{0}$ in $D$ arbitrarily.

Let $f(z)$ be the function which maps $D$ conformally on $P$. We denote by $\zeta_{k}$ the boundary point of $D$, which corresponds by this function to a vertex of $P$ with the interior angle $\alpha_{k} \pi$. If the vertex lies on the point at infinity, we agree to give $\alpha_{k}$ negative sign.

In the first place, zee assume that $P^{\circ}$ contains in it neither points of ramification nor points lying at infinity.

Then, $z f^{\prime}(z)$ is regular and does not vanish in $D$, and when $z$ moves 
on the boundary of $D$ in positive direction, the variations of its argument are as follows :

$$
\begin{aligned}
& d \operatorname{Arg} z f^{\prime}(z)=d \operatorname{Arg} \frac{d f(z)}{d \log z}=d \operatorname{Arg} d f(z)-d \operatorname{Arg} d \log z, \\
& d \operatorname{Arg} d f(z)=\left(1-\alpha_{k}\right) \pi \text { at } \zeta_{k} \text { and }=0 \text { elsewhere, } \\
& d \text { Arg } d \log z=-\pi \quad \text { at } s_{k} \text { and } \quad=0 \text { elsewhere. }
\end{aligned}
$$

Therefore, while defining the distribution $\sigma$ by

$$
\sigma\left(\zeta_{k}\right)=\frac{1-\alpha_{k}}{2}, \quad \sigma\left(s_{k}\right)=\frac{1}{2} \text { and } \sigma \equiv 0 \text { elsewhere, }
$$

we obtain by theorem 1 .

$$
z f^{\prime}(z)=z_{0} f^{\prime}\left(z_{0}\right) \prod_{k} \Omega\left(z, \zeta_{k}\right)^{\frac{1-a_{k}}{2}} \prod_{k} \Omega\left(z, s_{k}\right)^{\frac{1}{2}}
$$

Thus, we have the following expression for the mapping function.

$$
f(z)=z_{0} f^{\prime}\left(z_{0}\right) \int_{z_{0} k}^{z} \Pi_{k}\left(z, \zeta_{k}\right) \frac{1-\alpha_{k}}{2} \Pi \Omega\left(z, s_{k}\right)^{\frac{1}{2}} \frac{d z}{z}+f\left(z_{0}\right) .
$$

In the general case, we denote by $z_{k}$ the point of $D$ which corresponds to a point of ramification of $m_{k}$-th order $\left(m_{k}>0\right)$ on $P$ lying in the finite part of the plane, and by $z_{k}^{\prime}$ the point which corresponds to a point of ramification of $m_{k}^{\prime}$-th order $\left(m_{k}^{\prime} \geqq 0\right)$ lying at infinity.

Then, $z f^{\prime}(z)$ has a zero of $m_{k}$-th order at $z_{k}$ and a pole of $\left(m_{k}^{\prime}+2\right)$-th order at $z_{k}^{\prime}$. We can apply Theorem 1 to the function

$$
z \cdot f^{\prime}(z) \cdot \frac{\prod_{k} \Omega\left(z, z_{k}\right)^{m_{k}}}{\prod_{k} \Omega\left(z, z_{k}\right)^{m /{ }_{k}+2}}
$$

and, since we have

$$
d \operatorname{Arg} \boldsymbol{Q}\left(z, z^{*}\right) \equiv 0
$$

on the boundary of $D$, the distribution $\sigma$ can be so determined as before. Therefore, we have

$$
z f^{\prime}(z)=C_{1} \prod_{k} \Omega\left(z, \zeta_{k}\right)^{\frac{1-\alpha}{2}} \cdot \frac{\prod_{k} \Omega\left(z, z_{k}^{\prime}\right)^{m_{k}{ }^{\prime+2}}}{\prod_{k} \Omega\left(z, z_{k}\right)^{m_{k}}} \cdot \prod_{k} \Omega\left(z, s_{k}\right)^{\frac{1}{2}},
$$

$C_{1}$ being a suitable constant.

Thus, we obtain the following 
On conformal representation of multiply connected polygonal domain. 197

Theorem 2. The function which maps $D$ conformally on $P$ is given by

$$
f(z)=C_{1} \int_{k}^{z} \prod_{k} \Omega\left(z, \zeta_{k}\right) \frac{1-\alpha_{k}}{2} \cdot \frac{\prod_{k} \Omega\left(z, z_{k}^{\prime}\right)^{m_{k^{\prime}}+2}}{\prod_{k} \Omega\left(z, z_{k}\right)^{m_{k}}} \cdot \prod_{k} \Omega\left(z, s_{k}\right)^{\frac{1}{2}} \cdot \frac{d z}{z}+C_{2},
$$

where $C_{1}$ and $C_{2}$ are constants depending on position and magnitude of $P$ and on the lower bound of the integration.

Remark 1. If one of the points $z_{k}$ or $z_{k}^{\prime}$ coincides with $z_{0}$, we have to understand $\Omega\left(z, z_{0}\right)$ to be $\equiv 1$ in the above formula.

Remark 2. Though we have deduced Theorem 2 under the assumption $n \geqq 2$, it is also valid for $n=1$, as can be seen easily, if $D$ is the domain $|z|<R$ and $z_{0}$ is the origin $z=0$.

By the expressions for $\boldsymbol{\Omega}(z, \zeta)$ given in $\S 2$., we can easily see that, in case $P$ is schlicht, the formula of Theorem 2 coincides for $n=1$ with the ordinary Schwarz-Christoffel's formula, and for $n=2$ with the formula given by Mr. Y. Komatu ${ }^{5}$.

\section{Mathematical Institute, Tokyo University.}

\section{References.}

1) H. Weyl : Die Idee der Riemannschen Fläche, § 16. (1913).

2) H. Weyl: 1. c.

3) As for the expression of $\Omega$ in the general case in terms of Bergman's kernel function, cf. M. Ozawa : Some canonical conformal maps and representations, Kōdai Mathematical Seminar Reports No. 3 June 1950.

4) Y. Komatu : Darstellungen der in einem Kreisringe analytischen Funktionen nebst den Anwendungen auf konforme Abbildung über Polygonalringgebiete. Japanese Journ. of Math. Vol. 19, No. 2 (1945).

5) Y. Komatu : 1. c. 